\title{
Editorial: “Glycovirology”
}

\author{
Yasuo Suzuki
}

College of Life and Health Sciences, Chubu University, Health Science Hills, 1200 Matsumoto-cho, Kasugai-shi Aichi 487-8501 Japan

Diseases caused by bacteria, viruses, fungi and other parasites are major causes of death, disability, and social and economic disruption for millions of people. Emerging and reemerging infectious diseases caused by viruses are some of the most important diseases that must be controlled in the 21st century. Many viruses carry sugar chains which play a role in their escape from the host's immune systems and in their active transmission into the host. Host cell sugar chains are very diverse but the expression of each sugar chain in species, tissue, and individual cells is highly specific. In other words, natural sugar chains have very diverse structures but their expression is highly localized and specific. On the other hand, viruses have a defined host range specificity and recognize target host cells and receptor molecules on the host cell surface are highly specific. This fact suggests that viruses may have been taking advantage of the diversity of host sugar chains to expand the host range during evolution

*Address correspondence to this author at the Professor, College of Life and Health Sciences, Chubu University, Health Science Hills, 1200 Matsumotocho, Kasugai-shi, Aichi 487-8501, Japan; Tel/Fax: +81-568-51-6391;

E-mail: suzukiy@isc.chubu.ac.jp; and the high specificity of viruses for recognition of the target host cell receptor may reflect the highly specific expression of sugar chains by host cells.

The mutual recognition systems via sugar chains between viruses and their hosts may be deeply involved in the evolution of both viruses and their host animals. For this reason, the study of sugar chains should be promoted to control viral diseases, especially, viral glycobiology, a new research field, "glycovirology" covering both glycobiology and virology should be developed and extended globally. Carbohydrate/sugar chains have been shown to have important and diverse functions as receptors for viruses, as essential components of viruses, or as signal messengers in virus-infected cells. The sugar chains will surely be a very important target for research and drug discovery in this century because of its close relationship to viral infection. I would like to focus this issue to promote the research field of "glycovirology" 